\title{
Letalidad del cáncer de vesícula biliar es independiente del lugar de atención o características socio-demográficas. Chile 2002-2005
}

\author{
MAURICIO GABRIELLI ${ }^{1}$, SEBASTIÁN HUGO³, ANGÉLICA DOMÍNGUEZ², \\ SERGIO BAEZ ${ }^{4}$, ALIRO VENTURELLI ${ }^{5}$, MARÍA PUGA ${ }^{6}$, \\ ALFONSO DÍAZ ${ }^{3}$, NICOLÁS JARUFE ${ }^{1}$, CATTERINA FERRECCIO ${ }^{2}$
}

\section{Mortality due to gallbladder cancer. Retrospective analysis in three Chilean hospitals}

Background: Chile has the highest gallbladder cancer (GBC) death rate worldwide, affecting mainly Southern areas of the country. Aim: To compare the survival of GBC patients treated in hospitals located in areas with low and high risk for GBC. Material and Methods: Medical records of all patients with GBC admitted to one public hospital located in southern Chile, a public hospital and a private clinic, both located in Metropolitan Santiago, were reviewed. Cases were analyzed by age, sex, stage at diagnosis, ethnicity, socioeconomic status (SES) and rural residence. Survival was calculated using Kaplan Meier method. Results: A total of 598 cases (469 women), were analyzed. No differences in age or sex among hospitals were detected. At the moment of diagnosis, 75, 50 and 44\% of cases from the hospital in southern Chile, the public hospital in Santiago and the private clinic in Santiago, were in stage $I V$, respectively. Five years survival was lower in the public hospital in southern Chile than in the public hospital in Santiago (10.7 and 14.4\% respectively, $p<0.05)$ but not statistically different from the figure at the private clinic in Santiago (13.0\%). However, when adjusting for stage at the moment of diagnosis, no difference in survival between the three hospitals, was found. The median days of survival were 1,559, 188, 70 and 69 for stages I, II, III and IV respectively. Conclusions: GBC mortality is high. The stage at the moment of diagnosis is only significant predictor of survival.

(Rev Med Chile 2010; 138: 1357-1364).

Key words: Chile; Gallbladder neoplasms; Mortality; Survival rate.

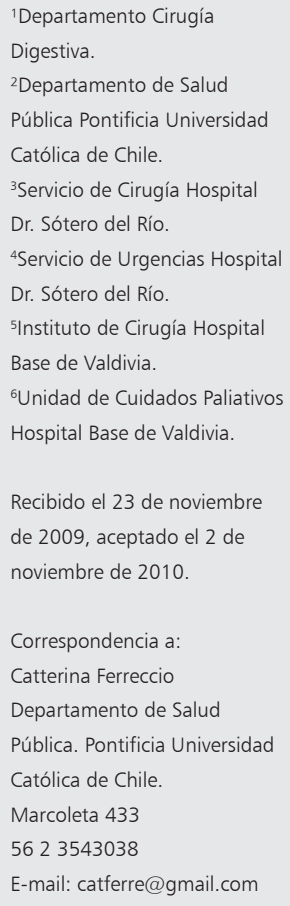

$\mathrm{E}$ 1 cáncer de vesícula biliar (CVB) es la primera causa de muerte oncológica en las mujeres chilenas. El año 2007 ocurrieron 1.905 muertes de CVB, 1.386 (72,8\%) en mujeres; la tasa de mortalidad por 100.000 habitantes fue 16,5 y 6,3 en mujeres y hombres respectivamente ${ }^{1}$; la mortalidad se ha mantenido en estos niveles desde $1990^{1}$. La distribución geográfica del CVB en Chile es heterogénea, con baja mortalidad, menor al promedio nacional, en las regiones del norte y centro del país, alcanzando las mayores tasas las regiones del sur; estas últimas tienen mayor pobreza y población mapuche que el resto de Chile ${ }^{2,3}$. Estudios nacionales e internacionales han informado la asociación entre el estadio tumoral al momento de la consulta y la sobrevida por $\mathrm{CVB}^{4-6}$. Sin embargo, se desconoce si hay diferencias de sobrevida al interior del país en relación a algunos factores socio-demográficos del huésped como ruralidad, el nivel socioeconómico (NSE) y la et- 
nia mapuche, o los recursos del lugar de atención, tienen un efecto independiente del estadio tumoral en la sobrevida por CVB. El objetivo del presente estudio fue comparar la sobrevida de pacientes con CVB atendidos en centros hospitalarios de zonas de bajo y de alto riesgo poblacional de CVB (Santiago y Valdivia respectivamente) ajustando por las características clínicas y socio-demográficas de los casos.

\section{Material y Método}

\section{Casos}

Se diseñó un estudio retrospectivo de todos los pacientes que ingresaron con el diagnóstico de CVB entre los años 2002 y 2005 al Hospital Regional de Valdivia (HBV), al Hospital Dr. Sótero del Río (HSR) y al Hospital Clínico de la Pontificia Universidad Católica de Chile (PUC). Se revisó la base de datos de cada centro obteniéndose información sobre la edad, sexo, fecha de diagnóstico y comuna de residencia; del Registro Civil se obtuvo información sobre los cuatro apellidos, fecha y lugar de nacimiento, fecha y causa de muerte. El diagnóstico de CVB en los tres hospitales se obtuvo por medio de ecotomografía abdominal, tomografía computada o biopsia. Se registró el estadio al momento del diagnóstico siguiendo la clasificación de la sexta edición de la American Joint Committee on Cancer? .

\section{Definiciones operacionales}

Se clasificó como NSE bajo a los casos de las categorías A o B según el Fondo Nacional de Salud (FONASA) y que además vivían en una comuna con $10 \%$ o más de población en situación de pobreza según la Encuesta de Caracterización Socioeconómica $2006^{8}$. Se clasificó a los sujetos con ascendencia mapuche cuando tenían al menos un apellido mapuche ${ }^{9}$. Como residentes de área rural se categorizó a los habitantes de comunas con menos de 150 habitantes $/ \mathrm{km}^{210}$. Se consideró como área geográfica de alto riesgo para CVB a la zona sur y de bajo riesgo a la zona central y norte de Chile, según la definición utilizada previamente por Andia y cols ${ }^{2}$.

\section{Sobrevida}

La fecha de cierre del estudio para efectos de cálculos de sobrevida actuarial fue el 31 agosto de
2009. Se calculó la sobrevida a tres y cinco años para CVB y se censuraron las muertes por otras causas. Se exploró la influencia del hospital de origen, estadio, sexo, ruralidad, NSE, edad al diagnóstico y ascendencia mapuche en la sobrevida de los casos con CVB. La sobrevida se calculó con el método de Kaplan Meier y las curvas para variables seleccionadas fueron comparadas usando las pruebas de Logrank ${ }^{11}$. Las variables asociadas significativamente a sobrevida se analizaron mediante un modelo multivariado de Cox, para identificar las que presentaban un efecto independiente en la sobrevida. El análisis estadístico se realizó con el programa SPSS16.0 $0^{12}$.

\section{Resultados}

\section{Características clínicas y demográficas}

En el período de estudio se hospitalizaron 598 casos con diagnóstico de CVB, 469 (78,4\%) mujeres, con una relación de 3,6 a 1 con respecto a los hombres. La edad media fue 63 años (rango de 28 a 101 años), 71 (11,9\%) de los casos tenía ascendencia mapuche, $467(78,1 \%)$ pertenecía al grupo de NSE bajo y $168(28,1 \%)$ vivía en una zona rural. Los casos fueron similares en su distribución por edad y sexo en los tres hospitales; sin embargo, los casos del HBV en comparación con los casos de Santiago, tenían significativamente mayor proporción de ruralidad, de bajo NSE y de ascendencia mapuche; también fue mayor la frecuencia de casos nacidos en el sur del país (regiones VI al sur) en el HBV comparado con HSR o la PUC (99\%, 54,3\% y 40\% respectivamente). Entre los casos del HBV fue significativamente mayor la frecuencia de estadio IV que entre los casos de la PUC o el HSR (77,3\%, 48,8\% y 53,0\% respectivamente) (Tabla 1 ).

\section{Causa de muerte de los casos del estudio}

De los 598 casos, $512(85,6 \%)$ habían muerto a la fecha de cierre, 495 por cáncer de vesícula biliar y 17 por otra causa. Entre estos últimos se registró como causa de muerte: otros cánceres (9), neumonía (3), accidente vascular encefálico (1), shock cardiogénico (1), daño hepático crónico (1) y paro cardio-respiratorio (2). Setenta y dos casos seguían vivos a agosto de 2009. En 14 (2,3\%) casos no se pudo determinar su estado, la mayoría de éstos eran del HBV $(\mathrm{n}=11)$. 
Tabla 1. Estadio y características demográficas cáncer de vesícula biliar, 2002-2005. Hospital Sótero del Río (HSR), Base Valdivia (HBV) y Clínico de la PUC (PUC)

\begin{tabular}{|lccc|}
\hline $\begin{array}{l}\text { Característica } \\
\text { pacientes }\end{array}$ & $\begin{array}{c}\text { HBV } \\
\text { (n: 264) }\end{array}$ & $\begin{array}{c}\text { PUC } \\
\text { (n: 91) }\end{array}$ & $\begin{array}{c}\text { HSR } \\
\text { (n: 243) }\end{array}$ \\
\hline $\begin{array}{l}\text { Estadio al diagnóstico** } \\
\text { I }\end{array}$ & $8,7 \%^{\mathrm{a}, \mathrm{b}}$ & $18,7 \%^{\mathrm{a}}$ & $22,6 \%^{\mathrm{b}}$ \\
II & $12,0 \%^{\mathrm{a}, \mathrm{b}}$ & $11,0 \%^{\mathrm{a}}$ & $14,4 \%^{\mathrm{b}}$ \\
III & $1,5 \%^{\mathrm{a}, \mathrm{b}}$ & $16,5 \%^{\mathrm{a}}$ & $7,4 \%^{\mathrm{b}}$ \\
IV & $75,0 \%^{\mathrm{a}, \mathrm{b}}$ & $44,0 \%^{\mathrm{a}}$ & $50,0 \%^{\mathrm{b}}$ \\
Estadios I-II & $20,7 \%^{\mathrm{a}, \mathrm{b}}$ & $29,7 \%^{\mathrm{a}}$ & $37,0 \%^{\mathrm{b}}$ \\
Mujeres & $79,2 \%$ & $76,0 \%$ & $78,6 \%$ \\
\hline Edad en años & 65,3 & 63,4 & 61,4 \\
(media, rango) & $(34-91)$ & $(28-93)$ & $(36-101)$ \\
Mapuches & $22,0 \%^{\mathrm{a}, \mathrm{b}}$ & $1,1 \% \%^{\mathrm{a}}$ & $4,9 \%^{\mathrm{b}}$ \\
\hline Nivel socioeconómico bajo & $84,0 \%^{\mathrm{a}}$ & $1,1 \%^{\mathrm{a}, \mathrm{c}}$ & $73,3 \%^{\mathrm{c}}$ \\
\hline Residencia Rural & $61,0 \%^{\mathrm{a}, \mathrm{b}}$ & $6,6 \%^{\mathrm{a}, \mathrm{c}}$ & $0,4 \% \%^{\mathrm{b}, \mathrm{c}}$ \\
\hline
\end{tabular}

*Comparación significativa entre Hospitales $(p<0,05)$ : $a=$ diferencia significativa entre HBV-PUC. $b=$ diferencia significativa entre HBV-HSR. $c=$ diferencia significativa entre PUC-HSR.

\section{Análisis de sobrevida}

Un año después del diagnóstico de CVB, había fallecido $20 \%$ de los casos en estadio I y $92 \%$ de los casos en estadio IV. A cinco años habían fallecido $40 \%$ de los casos en estadio I y $97 \%$ de los casos en estadio IV (Figura 2). La sobrevida global por CVB a cinco años fue significativamente menor en el HVB que en el HSR no alcanzando diferencia estadística con la PUC (Figura 1, Tabla 2); sin embargo, al ajustar por estadio no hubo diferencias significativas entre los hospitales de Santiago y Valdivia (Figura 3). En el análisis univariado de sobrevida en el HBV hubo significativamente menor sobrevida que en los hospitales de Santiago entre los casos de sexo femenino, los no mapuches, las personas de NSE bajo

Tabla 2. Comparación de sobrevida a 3 y 5 años de cáncer de vesícula biliar: 2002-2005. Hospitales Sótero del Río (HSR), Base Valdivia (HBV) y Clínico de la PUC (PUC)

\begin{tabular}{|c|c|c|c|c|c|c|c|}
\hline \multirow[b]{2}{*}{ Características (n) } & \multicolumn{3}{|c|}{ \% sobrevida a 3 años } & \multicolumn{3}{|c|}{ \% sobrevida a 5 años } & \multirow{2}{*}{$\begin{array}{l}\text { Diferencia curva } \\
\text { global de sobrevida* }\end{array}$} \\
\hline & $\begin{array}{l}\text { HBV } \\
(253)\end{array}$ & $\begin{array}{l}\text { PUC } \\
(90)\end{array}$ & $\begin{array}{l}\text { HSR } \\
(241)\end{array}$ & $\begin{array}{l}\text { HBV } \\
(253)\end{array}$ & $\begin{array}{l}\text { PUC } \\
(90)\end{array}$ & $\begin{array}{l}\text { HSR } \\
(241)\end{array}$ & \\
\hline Sobrevida global & 11,1 & 16,9 & 17,9 & 10,7 & 13,0 & 14,4 & $0,003^{b}$ \\
\hline \multicolumn{8}{|l|}{ Estadio** } \\
\hline I (95) & 82,6 & 64,7 & 58,2 & 78,3 & 58,8 & 49,4 & 0,232 \\
\hline II (72) & 13,3 & 22,2 & 3,1 & 13,3 & 11,1 & 3,1 & 0,050 \\
\hline III (37) & 0,0 & 0,0 & 0,0 & 0,0 & 0,0 & 0,0 & 0,572 \\
\hline IV (350) & 2,1 & 5,1 & 1,7 & 2,1 & 0,0 & 0,0 & 0,133 \\
\hline Mujeres (456) & 10,6 & 14,9 & 16,7 & 10,1 & 11,2 & 13,0 & $0,004^{b}$ \\
\hline Hombres (128) & 13,2 & 22,7 & 22,4 & 13,2 & 18,2 & 20,4 & 0,505 \\
\hline Mapuches (69) & 10,7 & 0,0 & 0,0 & 10,7 & 0,0 & 0,0 & $<0,001^{\mathrm{a}, \mathrm{c}}$ \\
\hline No mapuche (515) & 11,2 & 17,0 & 18,8 & 10,7 & 13,1 & 15,1 & $0,003^{b}$ \\
\hline NSE bajo (397) & 11,9 & 0,0 & 17,9 & 11,5 & 0,0 & 14,4 & $0,005^{b}$ \\
\hline NSE medio-alto (187) & 5,9 & 17,4 & - & 5,9 & 13,4 & - & 0,123 \\
\hline Rural (165) & 15,8 & 33,3 & 0,0 & 15,2 & 16,7 & 0,0 & 0,214 \\
\hline Urbano (419) & 3,2 & 15,7 & 18,2 & 3,2 & 12,7 & 14,6 & $<0,001^{a, b}$ \\
\hline
\end{tabular}

NSE: Nivel socioeconómico. **En 31 casos no se obtuvo el estadio. *: valor p comparación sobrevida a 5 años entre hospitales. Letras indican los pares que difieren significativamente: $a=$ diferencia significativa entre HBV-PUC. $b=$ diferencia significativa entre HBV-HSR. $c=$ diferencia significativa entre PUC-HSR 

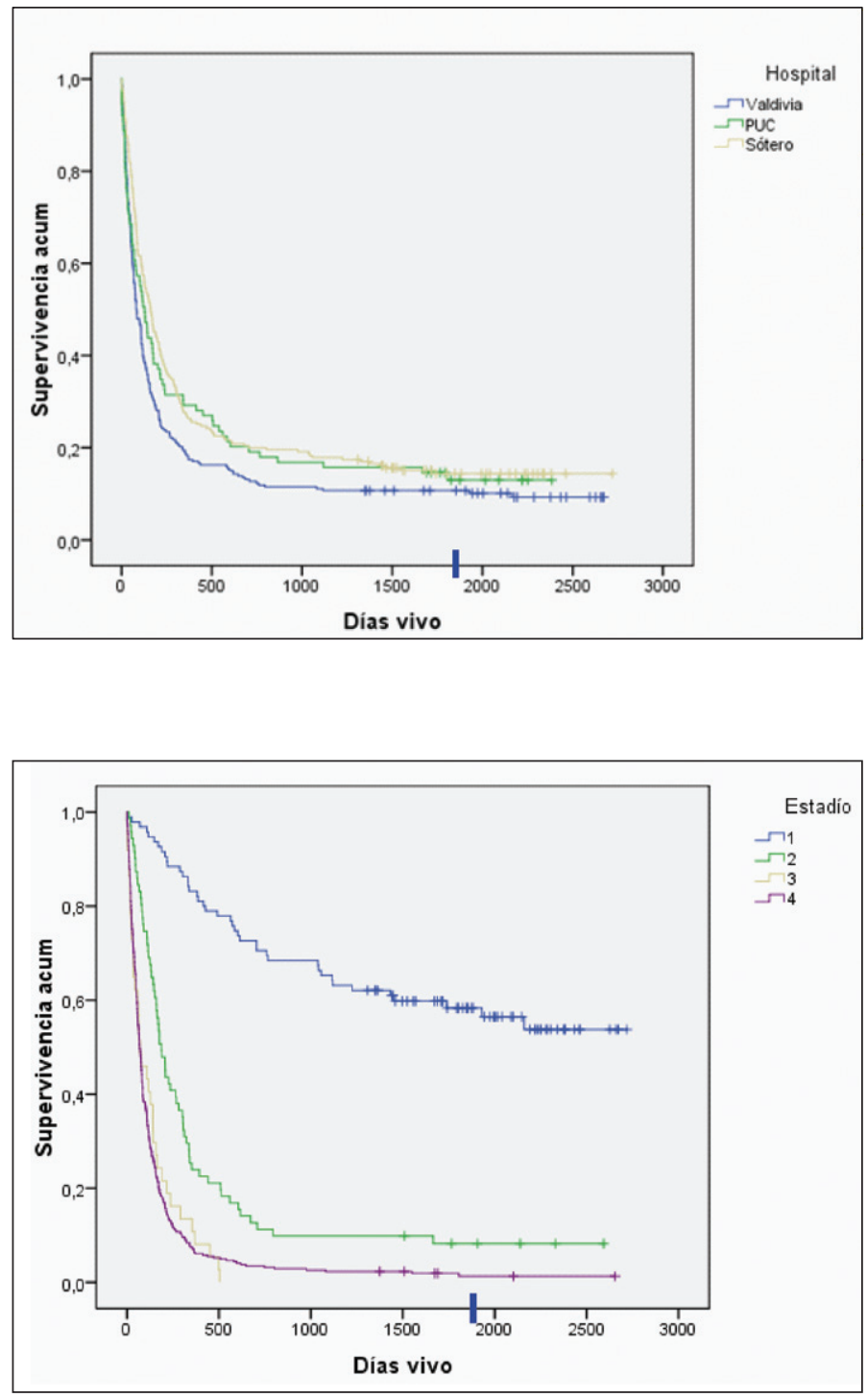

Figura 1. Sobrevida global por hospital de los 584 casos atendidos en el período $(p<0,01$ entre HBV y HSR).
Figura 2. Sobrevida por estadio de los 554 casos atendidos en los tres hospitales de Chile $(p<0,001)$. y los residentes urbanos (Tabla 2). Sin embargo, cuando se analizaron todos estos factores junto con hospital y estadio, mediante una regresión multivariada de Cox -donde la variable dependiente fue estar fallecido- la única variable significativa fue el estadio. En comparación con el estadio I, el riesgo relativo de morir a los 5 años fue 4,5 (95\% IC 3,0-6,7), 9,7 (95\% IC 6,0-15,4) y 9,5 (95\% IC $6,7-13,5)$ para los estadios II, III y IV respectivamente. La mediana de sobrevida en días de los casos fue 1.559, 188, 70 y 69 días para los estadios I, II, III y IV respectivamente.

\section{Discusión}

\section{Sobrevida}

En el presente estudio se confirmó que la gran mayoría de los casos de CVB habrán muerto a los cinco años del diagnóstico y que este mal pronóstico no mejora en un hospital con más recursos. Si bien el estadio más avanzado fue significativamente más frecuente en Valdivia, en los tres hospitales la sobrevida a tres años fue menor a $20 \%$ y a cinco años menor a $15 \%$. El hecho que la sobrevida por estadio fuera similar entre estos tres 

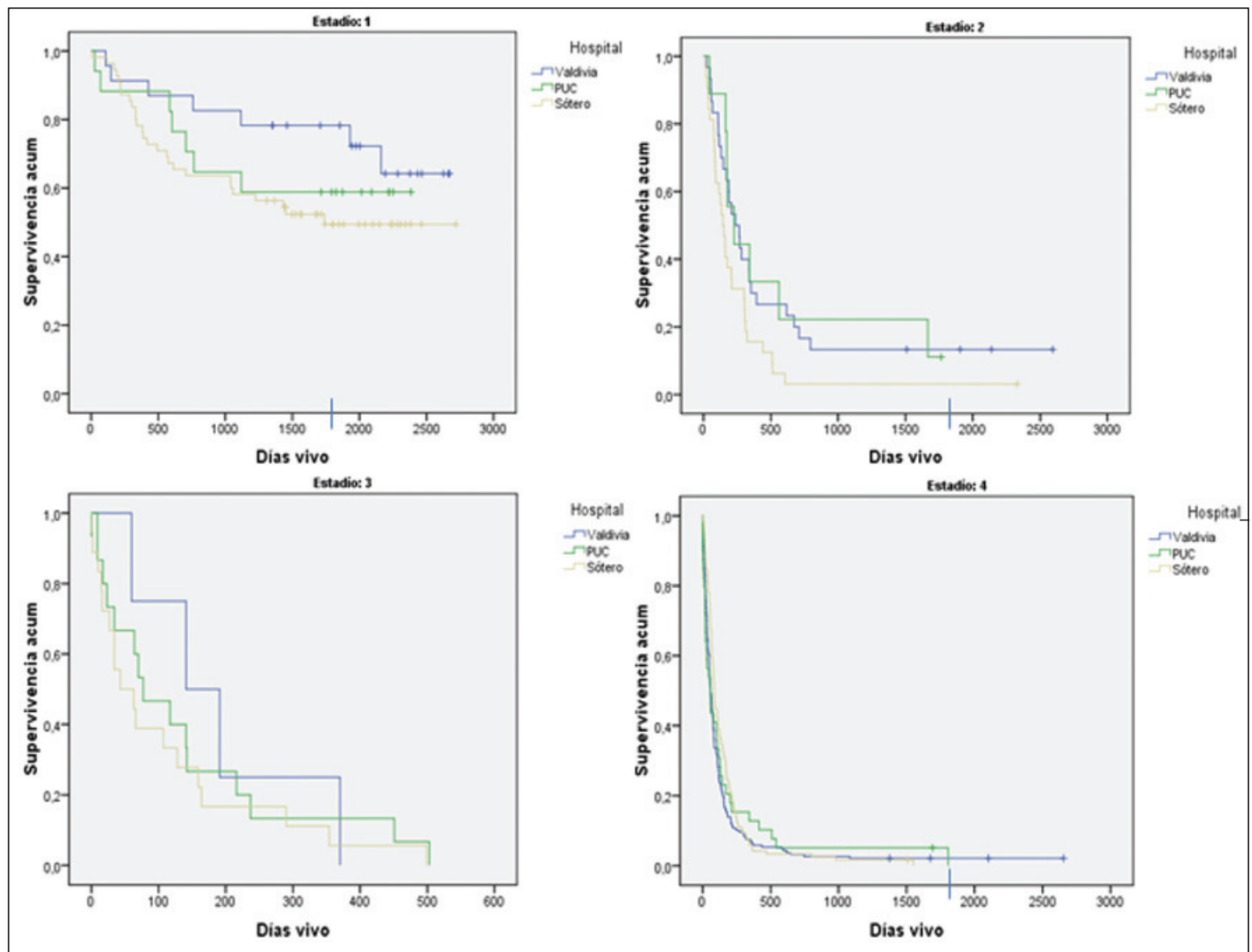

Figura 3. Sobrevida según estadio para cada uno de los hospitales del estudio ( $p=N S$ ).

hospitales con diferente nivel tecnológico, sugiere que el pronóstico del CVB no se ve influenciado por la oferta tecnológica del lugar de atención. Sólo el diagnóstico precoz logra mejorar el pronóstico de estos pacientes. Las diferencias que se observan en la sobrevida entre hospitales o según características de los pacientes (como etnia mapuche) se explican por las diferencias en el estadio en que llegan a consultar los pacientes. Este hallazgo es coincidente con otros estudios hospitalarios chilenos $^{4,5}$, así como con una reciente publicación de sobrevida de CVB de base poblacional7 ${ }^{7}$ La relativa independencia del pronóstico respecto de las acciones médicas ya había sido descrita por autores en Japón, quienes demostraron que ni resecciones quirúrgicas agresivas ni la quimioterapia modifican significativamente la sobrevida del $\mathrm{CVB}^{13}$. Otro estudio, también realizado en Japón y publicado recientemente, confirmó que la sobrevida de pacientes de CVB no cambia según el tipo de cirugía, la presencia de colelitiasis, la edad ni el sexo y que sólo está determinada por el estadio al momento del diagnóstico ${ }^{14}$. Tampoco existe aún terapia adyuvante que haya demostrado eficacia en el tratamiento del CVB ${ }^{15}$. La literatura internacional es clara en afirmar que la sobrevida de casos con CVB en estadio III y IV es de pocos meses, independiente del tipo de tratamiento que reciban ${ }^{16-18}$.

En el Hospital Clínico de la Pontificia Universidad Católica de Chile, según estadio se ofrece a los casos gemcitabina y cisplatino por un período de 4 a 6 meses, en los otros centros no se dispone de estos fármacos, utilizándose leucovorina y 5-fluorouracilo como drogas de primera línea. Sin embargo, Duffy y cols ${ }^{19}$ publicaron el año 2008 un estudio de 435 casos con CVB y no observaron beneficios en la sobrevida con terapia 
adyuvante. Asimismo, estudios chilenos mostraron que el pronóstico del CVB está determinado principalmente por la profundidad de invasión del tumor y es independiente del tipo de tratamiento, con una sobrevida global a 5 años de $78 \%$ para aquellos cánceres que no sobrepasaron la capa muscular y de $67,7 \%$ para aquellos circunscritos a la subserosa ${ }^{20,21}$. El riesgo de invasión linfática y de tumor residual luego de una cirugía aumenta con la profundidad de invasión del tumor ${ }^{22}$.

Pese a los avances de la medicina, específicamente en el campo quirúrgico en Chile, la sobrevida por CVB demostrada en este estudio es baja. Estos hallazgos sugieren que los esfuerzos de investigadores y administradores de la salud pública en nuestro país deben orientarse hacia la prevención. Se requieren estudios poblacionales para identificar las causas de la alta incidencia de este cáncer en Chile, debiendo explorar factores de riesgo locales, sea infecciones crónicas ${ }^{23}$ o factores asociados a la formación de cálculos biliares $^{24}$, factores alimentarios ${ }^{25} \mathrm{y}$ otros factores del huésped asociados al desarrollo de litiasis y de cáncer ${ }^{26}$. También es necesario evaluar en Chile la efectividad, aceptabilidad e impacto de estrategias de prevención primaria y secundaria dirigidas a grupos de alto riesgo. Se ha propuesto por ejemplo, realizar tamizajes con ecotomografías para identificar lesiones vesiculares y litiasis, seguidos de colecistectomía profiláctica ${ }^{27}$. Estas medidas deberían focalizarse en las zonas de mayor riesgo de CVB, para lograr un mayor beneficio en relación a los recursos.

El año 2006 el Ministerio de Salud de Chile inició un programa de prevención del CVB, que garantiza acceso a una colecistectomía a todo ciudadano chileno de 35 a 49 años de edad portador de una litiasis vesicular ${ }^{28}$. Sin embargo, en ese rango de edad sólo ocurre $10,7 \%$ de los cánceres incipientes en Chile ${ }^{29}, 90 \%$ de los cánceres incipientes se detectan en mayores de 49 años y $40,1 \%$ entre población económicamente activa de 50 a 64 años ${ }^{29}$. Aunque el programa ministerial tiene un claro fundamento en estudios de costoefectividad $^{30}$, tardaría muchos años en lograr un impacto de salud pública. En Temuco, Roa I ,en $2009^{31}$, entre 20.000 biopsias de mujeres colecistectomizados, muestra la edad media de pacientes con lesiones pre-cancerosas: displasia 50,9 años, carcinoma incipientes mucoso 55,6 años, incipiente musculares 60,9 años; confirmando que las edades medias de las mujeres con lesiones erradicables son mayores que las edades actualmente beneficiadas por el programa ministerial.

Para lograr una rápida mejoría en la situación del CVB en Chile, pensamos que se debieran dirigir nuevos recursos hacia las mujeres residentes en las áreas de mayor riesgo del sur de Chile y allí ampliar la edad hasta los 64 años. Esta intervención debiera basarse en colecistectomía laparoscópica, que actualmente representa 90\% de las colecistectomías en los países desarrolla$\operatorname{dos}^{32}$. La conveniencia de la focalización de una campaña sanitaria en las zonas de alto riesgo del sur de Chile se fundamenta la fuerte segregación espacial de este cáncer, ya descrita en publicaciones previas $^{2,3}$. Los registros poblacionales de cáncer de Valdivia y de Antofagasta -Sur y Norte de Chile respectivamente-, muestran que en el quinquenio 1998-2002, la incidencia de CVB en mujeres fue 4 veces mayor en Valdivia que en Antofagasta (26,2 y 5,4 casos por 100.000 mujeres respectivamente $)^{33}$. Esta segregación geográfica fue detectable también en nuestro estudio, donde confirmamos que la mayoría de los casos atendidos en Santiago nacieron en la zona sur de Chile. En el HSR, $35,4 \%$ de las mujeres con CVB nacieron en el sur de Chile, dos veces más que lo encontrado para mujeres atendidas en este hospital por un cáncer cervicouterino (17,7\% según C. Ferreccio comunicación personal).

\section{Limitaciones}

Una limitación metodológica del estudio fue la estimación del nivel socioeconómico de los casos, pues se utilizó la categoría según FONASA y el nivel de pobreza de la comuna de residencia como indicadores de $\mathrm{NSE}^{34}$. Cabe mencionar además, que el mestizaje en Chile es importante y que la ausencia de apellidos Mapuches no asegura que el paciente no sea portador de genes de esa población, ya que este mestizaje estaría presente en toda la geografía nacional ${ }^{35}$. Por otra parte, se trata de un estudio retrospectivo desde el punto de vista de recolección de la información, cuya principal limitación es que la información que se obtiene depende de los registros que se haya realizado. Afortunadamente, en nuestro estudio, las principales variables fueron recogidas sistemáticamente en los tres hospitales - registro de todos los casos ingresados con el diagnóstico de CVB, estadio, sexo, edad, número nacional de identifi- 
cación (RUT), intervenciones quirúrgicas, fechas de ingreso y alta y estado al alta-. Por otro lado, la información clave para la sobrevida -fecha y causa de la defunción- la obtuvimos en el registro civil que tiene prácticamente cobertura universal de las muertes. Con todo, hubo 14 casos $(2,3 \%)$ en quienes no se puedo determinar su estado al cierre del estudio, la mayoría de éstos proviene de Valdivia, no pudiéndose descartar algún problema en la notificación o certificación de las muertes en esa región.

En conclusión, sólo la detección precoz de cánceres incipientes puede mejorar la sobrevida y disminuir la mortalidad por este cáncer. Mientras el programa nacional alcanza la cobertura necesaria en los grupos jóvenes de todo el país, es urgente plantearse orientar recursos adicionales para las mujeres residentes en las zonas de más alto riesgo ampliando la edad hasta los 64 años de modo de detectar y tratar la mayoría de los cánceres incipientes en esa población.

Agradecimientos: Agradecemos a Claudia Bambs por su colaboración en la planificación del estudio.

\section{Referencias}

1. Ministerio de Salud. http://163.247.51.54/nuev/salida/ vesicula_1990_2007.php. revisado el 25 de Agosto 2010.

2. Andia M, Hsing A, Andreotti G, Ferreccio C. Geographic variation of gallbladder mortality and risk factors in Chile: a population-based ecologic study. Int J Cancer 2008; 123: 1411-16.

3. Andia M, Gederlini A, Ferreccio R. Cáncer de vesícula biliar: Tendencia y distribución del riesgo en Chile. Rev Med Chile 2006; 134: 565-74.

4. Venturelli F, Cárcamo C, Born M, Venegas L, Bertrán M, Venturelli A, et al. Cáncer vesicular en colecistectomías: Análisis de sobrevida y factores pronósticos. Rev Chilena de Cirugía 2008; 60: 277-81.

5. Roa I, de Aretxabala X, Araya J, Villaseca M, Roa J, Gilda $\mathrm{T}$, et al. Elementos morfológicos pronósticos en el cáncer de la vesícula biliar. Rev Med Chile 2002; 130: 387-95.

6. Bertran E, Heise K, Andia ME, Ferreccio C. Gallbladder Cancer: incidence and survival in a high-risk area of Chile. Int J Cancer 2010; 127: 2446-54.

7. Gallbladder. In: American Joint Committee on Cancer: AJCC Cancer Staging Manual. 6th ed. New York, NY: Springer, 2002; pp 139-44.
8. Ministerio de Planificación. Encuesta CASEN 2006. Disponible en www.mideplan.cl/casen/cpobreza_2006. html. Acceso 10 de Julio 2009.

9. Amigo H. Los Apellidos Indígenas. Relevancia, Limitaciones y Utilización. En Apellidos Mapuche. Historia y Significado. Santiago, Chile, 2008. Amigo H. Bustos P Editores. Editorial Maigret Ltda; ISBN:176970. Cap I: p. 13-21.

10. Organization for Economic Cooperation and Development (OECD) 1994. Disponible en www.oecd.com. Acceso 10 de Julio 2009.

11. Le CT. Applied Survival Analysis (Wiley Series Probability \&Mathematical Statistics). NJ:Wiley-Interscience, 1997.

12. Statistical Package for the Social Sciences for Windows. V16.0.1 Octubre 2007. Chicago Illinois.

13. Kayahara M, Nagakawa T. Recent Trends of Gallbladder Cancer in Japan. An Analysis of 4770 Patients. Cancer 2007; 110: 572-80.

14. Gourgiotis S, Kocher H, Solaini L, Yarollahi A, Tsiambas E, Salemis N. Gallbladder cancer. The American Journal of Surgery 2008; 196: 252-64.

15. Reid K, De la Medina A, Donohue J. Diagnosis and Surgical Management of Gallblader Cancer: A Review. J Gastrointest Surg 2007; 11: 671-81.

16. Lai ECH, Lau WY. Aggressive surgical resection for carcinoma of the gallblader. Aust NZJ Surg 2005; 75: 441-4.

17. Gold DG, Miller RC, Haddock MG, Gunderson LL, Quevedo F, Donohue JH, et al. Adjuvant therapy for gallbladder carcinoma: the Mayo Clinic Experience. Int J Radiat Oncol Biol Phys 2009; 75: 150-5.

18. Reddy SK, Clary BM. Surgical management of gallbladder cancer. Surg Oncol Clin N Am 2009; 18: 307-24.

19. Duffy A, Capanu M, Abou-Alfa GK, Huitzil D, Jarnagin W, Fong Y, et al. Gallbladder cancer (GBC): 10-year experience at Memorial Sloan-Kettering Cancer Centre (MSKCC). J Surg Oncol 2008; 98: 485-9.

20. de Aretxabala X, Roa I, Mora J, Pincheira O, Burgos L, Silva J. Management of gallbladder cancer with invasion of the muscular layer. Rev Med Chile 2004; 132: 183-8.

21. de Aretxabala X, Roa I, Burgos L, Losada H, Roa JC, Mora J, et al. Gallbladder cancer: an analysis of a series of 139 patients with invasion restricted to the subserosal layer. J Gastrointest Surg 2006; 10: 186-92.

22. Konstantinidis IT, Deshpande V, Genevay M, Berger D, Fernández-del Castillo C, Tanabe KK. Trends in presentation and survival for gallbladder cancer during a period of more than 4 decades: a single-institution experience. Arch Surg 2009; 144: 441-7.

23. Dutta U, Garg PK, Kumnar R, Tandon RK. Thyphoid carriers among patients with gallstones are at increased 
risk for carcinoma of the gallbladder. Am J Gastroenterol 2000; 95: 784-7.

24. Portincasa P, Di Ciaula A, Wang HH, Moschetta A, Wang DQ. Medicinal treatments of cholesterol gallstones: old, current and new perspectives. Curr Med Chem 2009; 16: 1531-42.

25. Rai A, Mohapatra SC, Shukla HS. Correlates between vegetable consumption and gallbladder cancer. Eur J Cancer Prev 2006; 15: 134-7.

26. Báez S, Tsuchiya Y, Calvo A, Pruyas M, Nakamura K, Kiyohara C, et al. Genetic variants involved in gallstone formation and capsaicin metabolism, and the risk of gallbladder cancer in Chilean women. World J Gastroenterol 2010; 16 (3): 372-8.

27. Sheth S, Bedford A, Chopra S. Primary gallbladder cancer: recognition of risk factors and the role of prophylactic cholecystectomy. Am J of Gastrol 2000; 95 (6): 1402-10.

28. Ministerio de Salud de Chile. Guía Clínica. Colecistectomía preventiva del cáncer de vesícula en adultos de 35 a 49 años sintomáticos. Hallado en http://www.redsalud. gov.cl/archivos/guiasges/vesicula.pdf. Visitado 1 de Octubre 2009.

29. Bertrán E. 2005. “Supervivencia de pacientes con Cáncer de vesícula biliar en la provincia de Valdivia quinquenio 1998-2002". Tesis para optar al grado de magister en salud pública de la Universidad de Chile. Disponible a texto completo en: http://www.google.cl/\#hl=es\&source $=\mathrm{hp} \& \mathrm{q}=$ tesis + enriqueta + bertran $\& \mathrm{aq}=\mathrm{f} \& \mathrm{aqi}=\& \mathrm{aq} \mathrm{l}=\& \mathrm{o}$ $\mathrm{q}=\& g s \_r f a i=\& f \mathrm{fp}=1$ beb1918d08d34cb. Visitado el $28 \mathrm{de}$ agosto 2010 .

30. Puschel K, Sullivan S, Montero J, Thompson B, Díaz A. Cost-effectiveness analysis of a preventive program for gallbladder disease in Chile. Rev Med Chile 2002; 130: 447-59.

31. Roa I, Muñoz S, Ibacache G, de Aretxabala X. Desde la displasia hasta el cáncer de la vesícula biliar. Rev Med Chile 2009; 137: 873-80.

32. Kaafarani HM, Smith TS, Neumayer L, Berger DH, Depalma RG, Itani KM. Trends, outcomes, and predictors of open and conversion to open cholecystectomy in Veterans Health Administration hospitals. Am J Surg 2010; 200 (1): 32-40.

33. Ministerio de Salud de Chile. Registro nacional de tumores. Hallado en http://epi.minsal.cl/epi/html/invest/ Informe\%20Valdivia\%20Cancer.pdf. Acceso 18 de julio de 2010.

34. Mora JG. Influencia del origen familiar en el acceso a la educación, en la obtención de empleos y en los salarios. En economía de la Educación; temas de estudio e investigación 1997; 22: 195-21.

35. Rocco P, Morales C, Moraga M, Miquel J, Nervi F, Llop $\mathrm{R}$, et al. Composición genética de la población chilena. Distribución de polimorfismos de DNA mitocondrial en grupos originarios y en la población mixta de Santiago. Rev Med Chile 2002; 130: 125-31. 